\title{
Sentence Syntax and Content in the Human Temporal Lobe: An fMRI Adaptation Study in Auditory and Visual Modalities
}

\author{
Anne-Dominique Devauchelle $\mathrm{e}^{1,2,3}$, Catherine Oppenheim ${ }^{4}$, \\ Luigi Rizzi ${ }^{5}$, Stanislas Dehaene ${ }^{1,2,6}$, and Christophe Pallier ${ }^{1,2,3}$
}

\begin{abstract}
Priming effects have been well documented in behavioral psycholinguistics experiments: The processing of a word or a sentence is typically facilitated when it shares lexico-semantic or syntactic features with a previously encountered stimulus. Here, we used fMRI priming to investigate which brain areas show adaptation to the repetition of a sentence's content or syntax. Participants read or listened to sentences organized in series which could or not share similar syntactic constructions and/or lexico-semantic content. The repetition of lexicosemantic content yielded adaptation in most of the temporal and frontal sentence processing network, both in the visual
\end{abstract}

\section{INTRODUCTION}

Understanding a sentence requires the interplay of phonological, lexical, syntactic, and semantic/pragmatic knowledge. It is generally assumed that a parser regroups individual words into phrases and incrementally builds a syntactic structure which enters into the computation of the semantic representation.

The functioning of the mental parser can be investigated by means of imaging techniques. Listening to or reading syntactically anomalous sentences elicits specific evoked brain potentials (Friederici, 2002; Osterhout \& Holcomb, 1992) and produces increased brain activations in inferior frontal and superior temporal areas (Friederici, Rüschemeyer, Hahne, \& Fiebach, 2003; Newman, Pancheva, Ozawa, Neville, \& Ullman, 2001). Other designs, besides the manipulation of syntactic violations, have been used to search for neural regions involved in syntactic processing, for instance, comparing the processing of matched syntactic and nonsyntactic stimuli (Indefrey, Hagoort, Herzog, Seitz, \& Brown, 2001; Friederici, Meyer, \& von Cramon, 2000; Mazoyer et al., 1993) and correlating brain

\footnotetext{
${ }^{1}$ INSERM, Gif/Yvette, France, ${ }^{2}$ CEA, DSV/I2BM, NeuroSpin Center, Gif/Yvette, France, ${ }^{3}$ Université Paris-Sud, Orsay, France, ${ }^{4}$ Université Paris-Descartes, Centre Hospitalier Sainte-Anne, Paris, France, ${ }^{5}$ CISCL, University of Siena, Siena, Italy, ${ }^{6}$ Collège de France, Paris, France
}

and the auditory modalities, even when the same lexicosemantic content was expressed using variable syntactic constructions. No fMRI adaptation effect was observed when the same syntactic construction was repeated. Yet behavioral priming was observed at both syntactic and semantic levels in a separate experiment where participants detected sentence endings. We discuss a number of possible explanations for the absence of syntactic priming in the fMRI experiments, including the possibility that the conglomerate of syntactic properties defining "a construction" is not an actual object assembled during parsing.

activity with grammatical complexity (Caplan, Alpert, \& Waters, 1999; Just, Carpenter, Keller, Eddy, \& Thulborn, 1996; Stromswold, Caplan, Alpert, \& Rauch, 1996). Brain imaging studies of syntax are reviewed by Grodzinsky and Friederici (2006) and Kaan and Swaab (2002). No clear conclusion has yet emerged about the locus of syntactic processing, probably in part because syntactic parsing is not a unitary process. In this article, we focus on syntactic trees and use syntactic priming as a tool to find the area(s) representing the syntactic structure associated with a sentence.

What is syntactic priming? In natural conversations, as well as in controlled language production tasks, people have a tendency to reuse recently encountered syntactic structures (see Pickering \& Branigan, 1999 for a review). For example, Bock (1986) found that participants are more likely to describe a picture using a passive sentence when they have just read a passive sentence rather than an active one. Using an on-line task, Smith and Wheeldon (2001) have observed that the latency to produce a sentence can also be decreased by syntactic priming. One interpretation of syntactic priming is that it reflects the fact that repeated use of procedures or representations reduces processing costs and increases processing speed (Levelt \& Kelter, 1982). Indeed, the repetition of a whole stimulus or of a feature of a stimulus often leads to a reduction in brain activity, a phenomenon which can be used to find the brain regions 
involved in processing this type of stimulus or feature (Dehaene-Lambertz et al., 2006; Grill-Spector \& Malach, 2001; Naccache \& Dehaene, 2001).

Early syntactic priming studies mostly used productionto-production priming. The effectiveness of priming supports the notion that the syntactic structure of the sentences is computed in speech production planning. Is it also computed in speech comprehension? A few studies demonstrated comprehension-to-production priming by having the participants hear or read the priming sentences before producing the target sentence (Bock, Dell, Chang, \& Onishi, 2007; Branigan, Pickering, Stewart, \& McLean, 2000; Potter \& Lombardi, 1998). These studies suggest a shared level of syntactic representation in comprehension and production, and therefore, predict the existence of syntactic priming in sentence comprehension.

However, the empirical data on comprehension-tocomprehension priming are actually very scarce: To our knowledge, only a few studies have investigated it (Branigan, Pickering, \& McLean, 2005; Noppeney \& Price, 2004; Frazier, Taft, Roeper, \& Clifton, 1984; Mehler \& Carey, 1967). In their study, Mehler and Carey (1967) presented participants with blocks of structurally homogeneous auditory sentences and found that the accuracy of perception of a sentence presented in white noise at the end of each block was improved if its phrasestructure was similar to that of the preceding sentences. A somewhat related design was used by Noppeney and Price (2004): Participants read a series of four sentences, which either had similar or dissimilar syntactic structures. Reading times were faster in the similar condition relative to the dissimilar condition. Moreover, Noppeney and Price used fMRI to scan their participants while reading covertly the same series of sentences. They found that when the syntax of sentences was repeated, brain activity was reduced in an area of the left anterior temporal lobe. This result fits with the proposal that the anterior part of the superior temporal lobe plays a role in phrase-structure building during sentence comprehension (Friederici, 2002; see also Mazoyer et al., 1993).

Noppeney and Price's (2004) materials included ambiguous and garden-path sentences arising from a small set of constructions. They used four types of sentences based on three different constructions (preposed adverbial clause, simple main clause, main clause with reduced relative). The present study also used an adaptation paradigm and fMRI. However, we departed from Noppeney and Price in several respects: (a) We exclusively used unambiguous sentences in order to abstract away from effects specifically linked to ambiguity resolution; (b) a broader variety of syntactic constructions was included; (c) we added series of sentences where the lexicosemantic content was repeated. More precisely, these sentences shared the same word forms (for content words), word meanings, and argument structure whereas the syntactic structures varied (see Table 1). This condi-
Table 1. The Four Experimental Conditions

Condition
Same sentences
Your work was ruined by a
trainee.
Your work was ruined by a
trainee.
Your work was ruined by a
trainee.
Your work was ruined by a
trainee.
It is this palace that the
architect designed.
It is a medicine that some
scientists invented.
It is your work that a trainee
ruined.
It is the rosebushes that my
gardener trampled
An architect designed this palace.
This palace was designed by an
architect.
It is this palace that the architect
designed.
By which architect was this
palace designed?
An architect designed this palace.
A medicine was invented by some
scientists.
It is your work that a trainee
Same content

In the same-syntax condition, the same syntactic construction and similar function words were used. In the same lexico-semantic content condition, the sentences involved the same open-class items (nouns, verbs, adjectives), and referred to the same event types and the same predicates and arguments, but this common lexico-semantic content was expressed by different syntactic constructions. In our experiment, the stimuli were in French.

tion was included to disentangle priming effects linked to the repetition of lexico-semantic content from syntactic priming effects (see Table 1). (d) The stimuli were presented either in the auditory or in the visual modalities (Noppeney and Price only used written sentences).

The questions of interest are the following: What brain regions, if any, will show reduction of activation when a syntactic tree is repeated? Likewise, what regions are affected by the repetition of lexico-semantic content? Are the regions involved in both cases similar, overlapping, or segregated? And finally, does input modality, auditory or visual, play a role? 


\section{EXPERIMENT 1}

\section{Methods}

\section{Participants}

Twenty-one young adults (13 women and 8 men; age: mean $=25$ years; $S D=4$; range $=18-33$ years) participated in the experiment. All were right-handed (scores at the Edinburgh test: mean $=77 ; S D=21$; range $=28$ 100) native speakers of French. The experiment was approved by the regional ethics committee, and written informed consent was obtained in all cases. The participants received $€ 75$ for their participation.

\section{Stimuli}

We created 76 sets of 16 sentences. Each set was obtained by crossing four kernel sentences with four syntactic structures (Table 2). Across all sets, a variety of syntactic constructions were employed : cleft sentences, wh-questions, relative clauses (reduced or not), yes/no questions, imperative, exclamative clauses, raising construction, preposed adverbial clauses, sentential subject sentences, simple ac-

Table 2. Example of One Set of Sixteen Sentences by Crossing Four Kernel Sentences (1, 2, 3, and 4) with Four Syntactic Structures (a, b, c, and d)

1a An architect designed this palace.

1b This palace was designed by an architect.

1c It is this palace that the architect designed.

1d By which architect was this palace designed?

2a Some scientists invented a medicine.

$2 \mathrm{~b} \quad$ A medicine was invented by some scientists.

2c It is a medicine that some scientists invented.

$2 \mathrm{~d} \quad$ By which scientists was this medicine invented?

3a A trainee ruined your work.

$3 \mathrm{~b} \quad$ Your work was ruined by a trainee.

3c It is your work that a trainee ruined.

3d By which trainee was your work ruined?

4a My gardener trampled the rosebushes.

$4 \mathrm{~b} \quad$ The rosebushes were trampled by my gardener.

4c These are the rosebushes that my gardener trampled.

4d By which gardener were the rosebushes trampled?

Across all sets, a variety of syntactic constructions were employed: cleft sentences, wh-questions, relative clauses (reduced or not), yes/no questions, imperative, exclamative clauses, raising construction, preposed adverbial clauses, sentential subject sentences, simple active clauses, and simple passive clauses. tive clauses, and simple passive clauses (the full list of stimuli is available at www.unicog.org/publications/Syntactic_ Adaptation_Experiments_Materials.html).

This material was used to create different experimental lists for each participant. These lists comprised 120 miniblocks of four sentences each: 30 miniblocks for each of the four experimental conditions: same sentences, same syntax, same lexico-semantic content, and different sentences (see Table 1). For each participant, a given sentence never occurred in more than one miniblock throughout the whole experiment. This was achieved by sampling sentences from the 76 sets. For example, to create a miniblock condition "same sentences," one set was randomly selected and then one sentence was randomly selected; this set was then discarded and not reused for any other miniblock. For the condition "same syntax," a set was randomly selected and four sentences with the same structure were extracted. With this procedure, a given sentence had the same likelihood of appearing in any of the four experimental conditions across subjects.

The sentences were digitally recorded in a quiet room by a female speaker. They were edited and saved with a resolution of 16 bits and a sampling frequency of $22.05 \mathrm{kHz}$. The sentences varied in length from 5 to 17 words and their durations when read aloud varied from 1.4 to $4.6 \mathrm{sec}$.

\section{Procedure}

Each participant was scanned in six sessions: three in which the stimuli were presented auditorily and three in which the stimuli were presented visually (the order was interleaved). Each session lasted $8 \mathrm{~min} 24 \mathrm{sec}$ and comprised 20 miniblocks of four sentences (plus a few additional sentences, see below). Sentences within a miniblock were separated by 2 sec of silence and each miniblock was separated from the other by $8 \mathrm{sec}$ of silence.

To induce the participants to attend to the stimuli, 30 probe sentences were inserted at random locations between miniblocks, which instructed them to press a button. Before entering the scanner, the participants were familiarized with the task with a short training of 18 sentences in each modality (including each condition and a few probe sentences instructing them to press a button).

The audio stimuli were delivered through headphones (Resonance Technologies, Northridge, CA). The visual stimuli were projected in rapid serial presentation at the rate of one word every 270 msec to make it comparable to the auditory condition.

\section{Scanning and Data Analysis}

Images were acquired with a 3-T Bruker Medspec 30/100 scanner. Functional images used EPI sequences $(\mathrm{TR}=$ $2.4 \mathrm{sec}, \mathrm{TE}=30 \mathrm{msec}$, matrix $=64 \times 64$, voxel size $=3 \times$ 
$3 \times 5 \mathrm{~mm}$, number of slices $=24$ ). The anatomical scan used a 3-D gradient-echo sequence $(\mathrm{TI}=2530 \mathrm{msec}$, $\mathrm{TE}=3.3 \mathrm{msec}, \mathrm{TR}=8.6 \mathrm{sec}$, voxel size $=1.33 \times 1.2 \times$

\section{$1.2 \mathrm{~mm})$.}

Data were processed with the SPM2 software. The first four volumes of each functional session were discarded and the remaining images were corrected for slicetiming differences and motion. The anatomical images were normalized using the MNI T1 template and the normalization parameters were applied to the functional images, which were finally smoothed with a $5 \mathrm{~mm}^{3}$ Gaussian kernel. For each subject, a general linear model was created which included, for each session, 16 independent variables obtained by crossing the factor "position" of the sentences within the miniblock $\left(1^{\text {st }}\right.$, $2^{\text {nd }}, 3^{\text {rd }}$, or $4^{\text {th }}$ ), and the factor "condition" with four levels (see Table 1). The event-related regressors used the canonical hemodynamic responses included in SPM2. Additional event-related regressors modeling the probe sentences and the associated motor responses were included.

For second-level group analyses (random-effect), a smoothing kernel of $8 \mathrm{~mm}^{3}$ was applied to the individual contrast images. One participant was detected as an out- lier using the Distance toolbox (Mériaux et al., 2003) and was excluded from further analyses.

\section{Results}

The probe sentences were detected with an accuracy of 94\%, showing that the participants attended to the stimuli. The first analyses of fMRI data looked for brain area showing a decrease in the BOLD response over the course of the presentation of the four sentences, using a linear contrast with the weights $(3,1,-1,-3)$ to estimate the slope of the BOLD response. These analyses were performed within the regions that were activated relative to silence (in the auditory modality) or fixation (in the visual modality). Figure 1 shows the results of these group analyses, split by "modality" (auditory or visual) and "condition." Notice that there was some adaptation even in the "different sentences" condition, primarily in unimodal sensory areas (Figure 1, last row).

In order to remove nonspecific adaptation effects in subsequent analyses, the different sentences condition was considered as a baseline and was subtracted from the other conditions. The results of the comparison

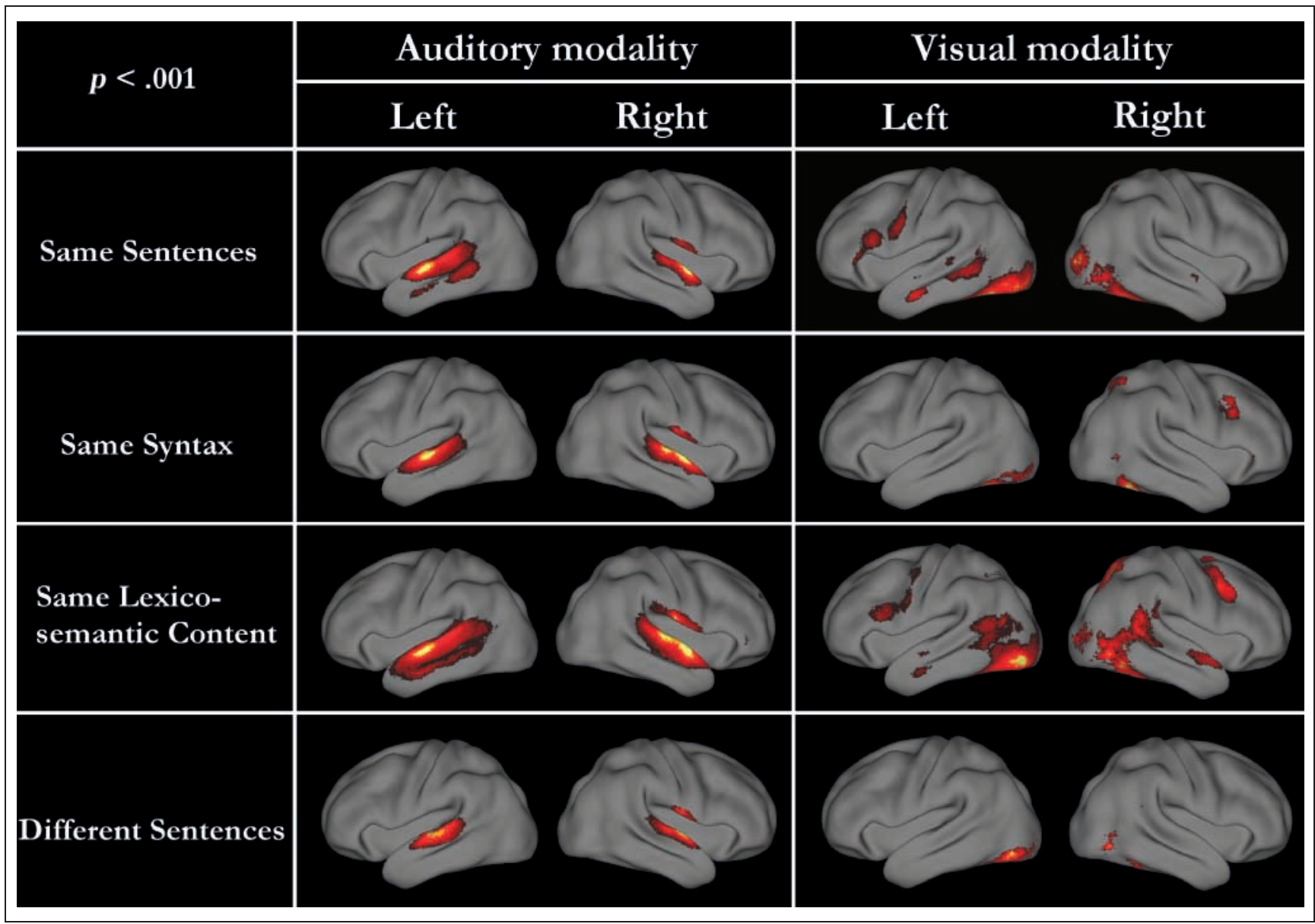

Figure 1. Areas showing a significant decrease in activation across the four sentences, as a function of condition and modality $(p<.001$, uncorrected voxel-based, $p<.05$ for extent). 


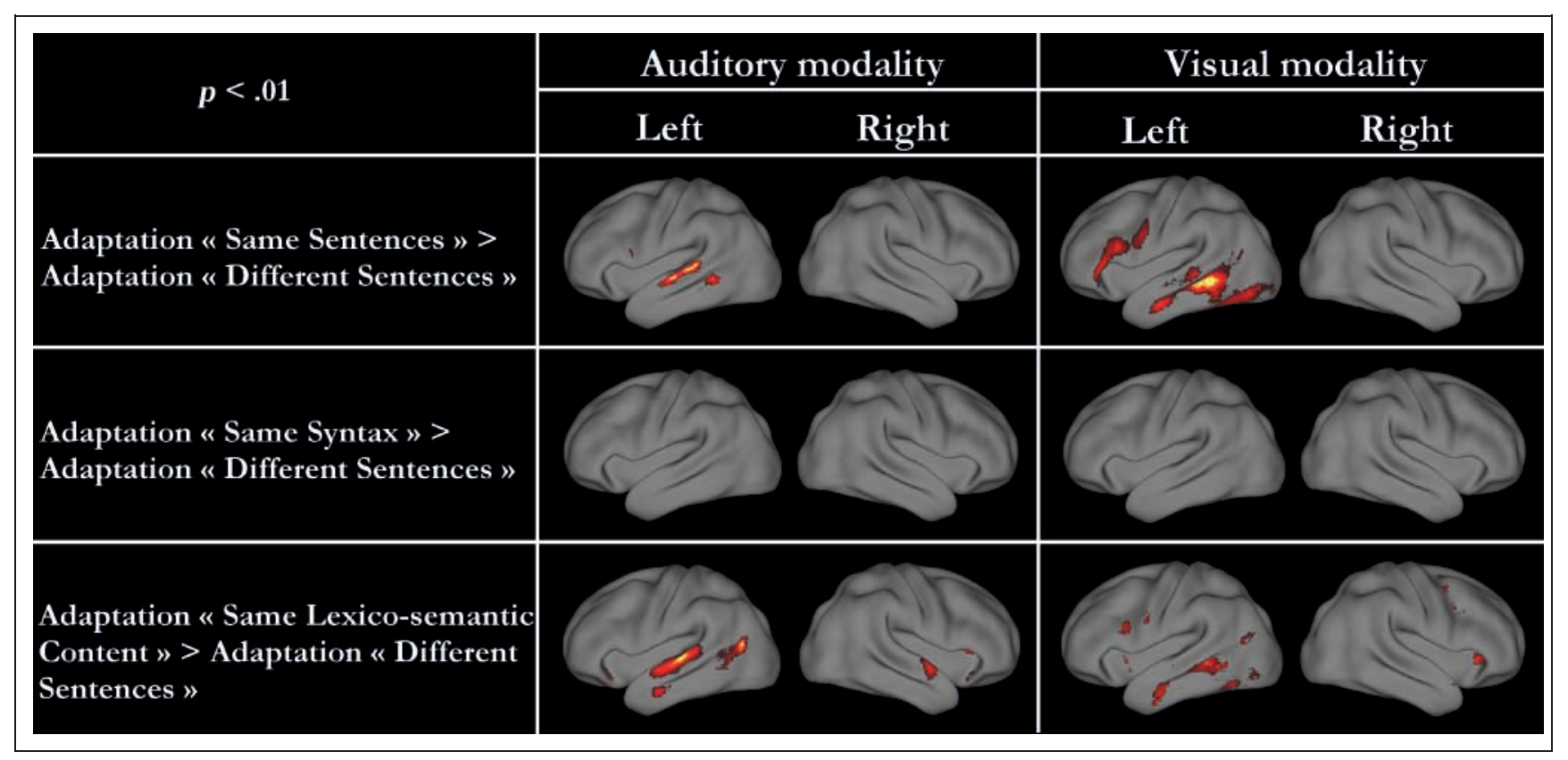

Figure 2. Areas showing more adaptation than in the "different-sentences" condition $(p<.01$, uncorrected voxel-based).

between each of these three conditions and the different sentences condition are displayed on Figure 2 and detailed in Table 3.

The time courses of the fMRI responses in several regions of interest (ROIs) are shown in Figure 3B. These ROIs are spheres of $8 \mathrm{~mm}$ of radius centered on the maxima of the maps displayed in Figure 2. Two additional ROIs were included: The first one, located in the left anterior temporal cortex, is centered on the MNI coordinates $(-42,4,-28)$ where syntactic adaptation was reported by Noppeney and Price (2004); The second one, located more dorsally in the superior temporal sulcus (STS; MNI coordinates: $-60,-8-4$ ), corresponded to the putative homologue of the visual word form area (VWFA) in the auditory stream according to Cohen, Jobert, Bihan, and Dehaene (2004).

When comparing the "same-syntax" condition to the "different-sentences" condition, no significant effect of adaptation emerged (even when the voxel-based statistical threshold was lowered to $p<.01$, uncorrected, either in the visual or in the auditory modality. The data were also analyzed using a $2 \times 2 \times 2$ analysis of variance with factors of modality, syntactic repetition (yes/no), and lexico-semantic content repetition (yes/no) to benefit from the added power of pooling across the entire dataset. Even with this analysis, we did not observe any significant effect of syntactic priming. In the specific ROI where Noppeney and Price found maximal adaptation to syntax (MNI coordinates: $-424-28$ ), the effect of lexico-semantic content repetition was significant at $p<.01$, uncorrected voxel-based, but there was no effect of repetition of syntactic structure. The plots of signal time course displayed in Figure 3B show that the effect of lexico-semantic content in the ROI $(-42,4$,
-28 ) is actually due to a relative increase of activation, in the auditory modality, when sentences do not share the same content words.

For the "same-sentence" condition, in the visual modality, adaptation effects were observed in the left inferior and middle temporal gyri, in the left inferior and middle occipital gyri, in the left fusiform gyrus, and in the left inferior frontal regions. In the auditory modality, adaptation effect was found in the left middle temporal gyrus and in the right cerebellum. All of these regions passed the false discovery rate (FDR) threshold of $p<.05$.

For the "same lexico-semantic content" condition, in the visual modality, adaptation effects were observed in the left middle temporal gyrus and in the left precentral gyrus. In the auditory modality, adaptation effect was found in the left middle and superior temporal gyri and in the right middle temporal gyrus. For the auditory modality, these regions passed the FDR threshold of $p<.05$, whereas in the visual modality, the clusters peaks had marginal FDR $p$ values of .12 .

A direct comparison between the "same-sentences" condition and the "same lexico-semantic" condition showed that the adaptations were not significantly stronger in the former condition than in the latter one.

The time courses of signal displayed in Figure 3B show that adaptation sometimes had a linear profile (e.g., see voxel $-44-60,-20$ in the visual condition) and sometimes a rather more logarithmic profile with most of the adaptation taking place between Sentence 1 and Sentence 2 (e.g., voxel $-60-12-8$, in the auditory modality). The previous set of analyses was replicated using a "logarithmic" contrast $(3-1-1-1)$ rather than a linear contrast $(31-1-3)$; these new analyses yielded similar patterns of adaptations than the previous one. 
Table 3. Peak Voxels of Activated Regions Showing Significant Adaptations $(p<.001$, Uncorrected Voxel-based; Clusters' Extent $>4$ Voxels; Masked by the "Sentence-Silence" Contrast at $p<.001$ )

\begin{tabular}{|c|c|c|c|c|c|c|}
\hline \multirow[b]{2}{*}{ Contrast } & \multirow[b]{2}{*}{ Z Score } & \multirow[b]{2}{*}{$p(F D R)$} & \multirow[b]{2}{*}{ Extent $\left(\mathrm{mm}^{3}\right)$} & \multicolumn{3}{|c|}{ MNI Coordinates } \\
\hline & & & & $x$ & $y$ & $z$ \\
\hline \multicolumn{7}{|c|}{ Adaptation Same Sentences > Adaptation Different Sentences } \\
\hline \multicolumn{7}{|l|}{ Auditory modality } \\
\hline Left middle temporal gyrus & 3.41 & .032 & 21 & -64 & -40 & 0 \\
\hline Left middle temporal gyrus & 3.38 & .032 & 33 & -64 & -20 & -4 \\
\hline Left middle temporal gyrus & 3.34 & .032 & & -60 & -12 & -8 \\
\hline Right cerebellum & 3.47 & .032 & 54 & 20 & -72 & -40 \\
\hline \multicolumn{7}{|l|}{ Visual modality } \\
\hline Left middle temporal gyrus & 4.46 & .008 & 81 & -60 & -12 & -16 \\
\hline Left middle temporal gyrus & 4.21 & .008 & 375 & -52 & -40 & 0 \\
\hline Left inferior temporal gyrus & 3.93 & .008 & & -44 & -44 & -16 \\
\hline Left inferior temporal gyrus & 3.92 & .008 & & -44 & -52 & -8 \\
\hline Left inferior occipital gyrus & 3.67 & .010 & & -44 & -60 & -16 \\
\hline Left fusiform gyrus & 3.62 & .010 & & -40 & -56 & -20 \\
\hline Left middle occipital gyrus & 3.42 & .012 & & -44 & -72 & -12 \\
\hline Left inferior frontal gyrus pars opercularis & 4.09 & .008 & 81 & -60 & 12 & 28 \\
\hline Left inferior frontal gyrus pars triangularis & 3.49 & .011 & 63 & -52 & 24 & 0 \\
\hline \multicolumn{7}{|c|}{ Adaptation Same Lexico-semantic Content $>$ Adaptation Different Sentences } \\
\hline \multicolumn{7}{|l|}{ Auditory modality } \\
\hline Left middle temporal gyrus & 3.49 & .028 & 36 & -60 & -12 & -8 \\
\hline Left superior temporal gyrus & 3.29 & .028 & 45 & -64 & -24 & 0 \\
\hline Left middle temporal gyrus & 3.26 & .028 & & -68 & -40 & 4 \\
\hline Right middle temporal gyrus & 3.28 & .028 & 12 & 60 & -8 & -12 \\
\hline \multicolumn{7}{|l|}{ Visual modality } \\
\hline Left middle temporal gyrus & 3.58 & .118 & 18 & -44 & -48 & 0 \\
\hline Left precentral gyrus & 3.37 & .118 & 12 & -56 & -4 & 36 \\
\hline
\end{tabular}

Figure $3 \mathrm{~A}$ shows the areas involved while reading (blue), listening (green), or in both modalities (red) on three contrasts: "sentences versus silence," "adaptation to the same sentences vs. adaptation to different sentence," and "adaptation to sentences with the same lexico-semantic content vs. adaptation to different sentence." In the contrast "sentences versus silence," common activations were located in the left superior and middle temporal gyri and in regions bordering the left STS. They were also found in the left inferior frontal regions and in the left precentral gyrus. The activations were more extended in the temporal gyrus for the auditory modality than for the visual modality, whereas the converse was true in the frontal and occipital regions. The temporal lobe regions showing adaptation in the auditory modality tended to be located more dorsally than those showing adaptation effects in the visual modality.

\section{Discussion}

The aim of the experiment was to highlight the areas encoding the syntactic structure and the lexico-semantic content of heard or read sentences. Our rationale was that such areas should show a decrease in activation when successive sentences sharing the same syntactic construction or the same lexico-semantic content were processed. Our results confirm previous findings that a large portion of the left STS and the inferior frontal language processing network is sensitive to sentence 


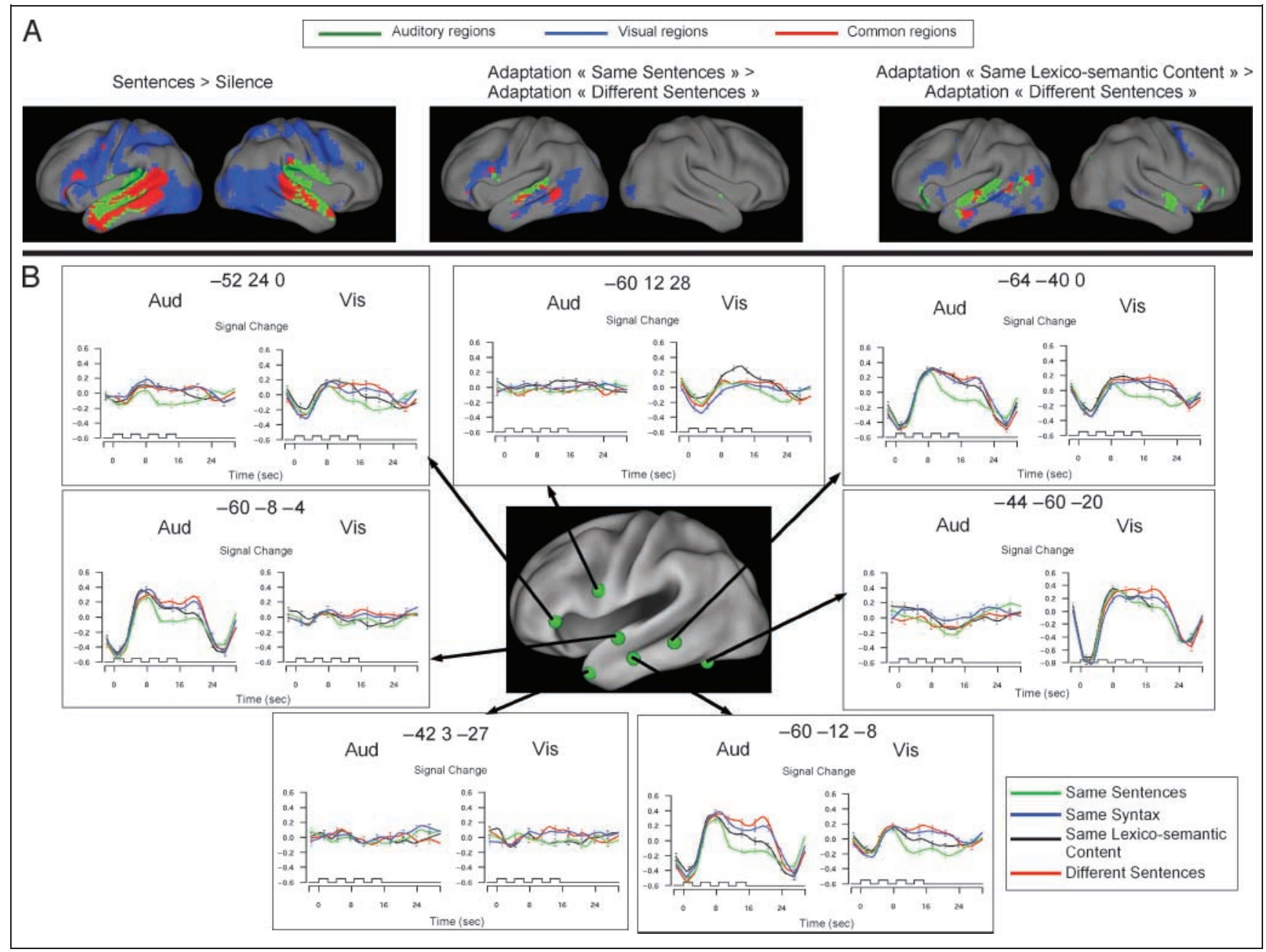

Figure 3. (A) Areas involved in reading (blue), listening (green), and their intersection (red) (contrast maps threshold at $p<.01$ for each modality). (B) Time course in regions of interest defined as spheres of $8 \mathrm{~mm}$ radius centered on the peaks defined by SPM shown in Figure 2 . Green line $=$ same-sentences adaptation; blue line $=$ same-syntax adaptation; black line $=$ same lexico-semantic content adaptation; red line $=$ different-sentences adaptation.

repetition (Dehaene-Lambertz et al., 2006; Hasson, Nusbaum, \& Small, 2006), thus vindicating the use of fMRI adaptation as a method to study the levels of representation in language processing. One novel finding is that adaptation can be observed even when the word order differs in successive sentences, but the lexico-semantic content is otherwise preserved.

Another one of our goals was to investigate the cerebral correlates of syntactic representation of sentences by examining which brain areas show reduced activation when sentences having the same syntactic structures are repeated. However, unexpectedly, no such area was observed either in the visual or in the auditory modality.

This absence of syntactic adaptation is at odds with the observation of Noppeney and Price (2004), who described a syntactic adaptation effect in a region of the left anterior temporal gyrus. As mentioned in the Introduction, our study differs from Noppeney and Price's on a number of points. Among others was the fact that although they used only four structures (late or early closure and simple active or reduced relative), our stimuli encompassed a much broader set of structures, most of which have not been previously tested in the syntactic priming literature. It is possible that syntactic priming works better with some types of constructions than others. We therefore decided to design a second syntactic priming experiment using only four constructions: sentences in active, passive, cleft, or cleft and passive forms. This new experiment would allow us to compare syntactic priming separately for each of these four constructions.

\section{EXPERIMENT 2}

\section{Methods}

\section{Participants}

Twenty young adults ( 7 women and 13 men; age mean $=$ 22 years; $S D=2$; range $=19-25$ years) participated in 
the experiment. All were right-handed (scores on the Edinburgh test: mean $=83 ; S D=19$; range $=30-100$ ) native speakers of French. The experiment was approved by the regional ethics committee, and written informed consent was obtained in all cases. The participants received $€ 80$ for their participation.

\section{Stimuli}

Contrary to Experiment 1, the sentences created for this experiment could only have one of four types of syntactic structures: active form (e.g., The blue car bit the small dog), passive form (e.g., The small dog was bit by the car), cleft form (e.g., It is the car that hit the small dog), and cleft-passive form (e.g., It is the dog that was bit by the car). Lists of 384 sentences organized in 96 miniblocks of four sentences were generated for each participant using a procedure similar to that of Experiment 1 (the full list of stimuli is available at www.unicog. org/publications/Syntactic_Adaptation_Experiments_ Materials.html). Half of the miniblocks belonged to the same-syntax condition, that is, the sentences expressed the same syntactic construction. A fourth of the miniblocks belonged to the same-sentence condition, and another fourth to the different-sentences condition. Each syntactic structure appeared equally often in the three experimental conditions. The length of the sentences in words varied from 8 to 10 .

\section{Procedure}

Each participant was scanned in four visual sessions. Each session comprised 24 miniblocks and lasted $10 \mathrm{~min}$. Sentences within miniblocks were separated by 2 sec of silence and the interval between them lasted 8 sec.

To induce participants to attend the stimuli, they were required to perform a recognition task after each session: 10 sentences were presented to the subject, who had to decide if the sentence was present in the previous session, by pressing the right or left button. Before entering the scanner, the participants were familiarized with the task with a short training of 16 sentences (including each condition) and a task training of 9 sentences.

The visual stimuli were projected in rapid serial presentation at the rate of one word every $270 \mathrm{msec}$.

\section{Scanning and Data Analysis}

Images were acquired with a 3-T Bruker Medspec 30/100 scanner. Functional images used EPI sequences $(\mathrm{TR}=$ $2.4 \mathrm{sec}, \mathrm{TE}=30 \mathrm{msec}$, matrix $=64 \times 64$, voxel size $=3 \times$ $3 \times 3 \mathrm{~mm}$, number of slices $=40$ ). The anatomical scan used a 3-D gradient-echo sequence $(\mathrm{TI}=2530 \mathrm{msec}$, $\mathrm{TE}=3.3 \mathrm{msec}, \mathrm{TR}=8.6 \mathrm{sec}$, voxels size $=1.33 \times 1.2 \times$ $1.2 \mathrm{~mm})$.
Data were processed with the SPM2 software. The first four volumes of each functional session were discarded and the remaining images were corrected for slicetiming differences and motion. The anatomical images were normalized using the MNI T1 template and the normalization parameters were applied to the functional images, which were finally smoothed with a $5 \mathrm{~mm}^{3}$ Gaussian kernel. For each subject, a general linear model was created, which included, for each session, 24 independent variables obtained by crossing the factor "position" of the sentences within the miniblock $\left(1^{\text {st }}\right.$, $2^{\text {nd }}, 3^{\text {rd }}$, or $\left.4^{\text {th }}\right)$, and the factor "condition" with three levels (same sentence, same syntax, different sentence), and only for the same-syntax condition did the factor syntactic structure have four levels (active, passive, cleft, and cleft-passive form). The event-related regressors used the canonical hemodynamic responses included in SPM2.

For second-level group analyses (random-effect), a smoothing kernel of $8 \mathrm{~mm}^{3}$ was applied to the individual contrast images.

\section{Results}

During the recognition sentence task, subjects recognized the sentence with an accuracy of $77 \%$, showing that the participants attended to the stimuli. The first analyses of fMRI data looked for brain areas showing a decrease in the BOLD response over the course of the presentation of the four sentences [using a linear contrast with the weights $(3,1,-1,-3)$ to estimate the slope of the BOLD response]. The results of these group analyses show that there was some adaptation even in the "different-sentences" condition, primarily in the unimodal sensory areas, as in the first experiment. Therefore, in order to remove nonspecific adaptation effects, the "different-sentences" condition was considered as a baseline and was subtracted from the three other conditions in subsequent analyses.

Table 4 lists the areas where the adaptation was stronger in the "same-sentences" than in the "differentsentences" condition (see also Figure 4), masked by the "sentence-fixation" contrast at $p<.001$. They include the left inferior occipital gyrus, the fusiform gyrus, the left inferior/middle temporal gyrus, and the frontal regions extending from the frontal operculum to the precentral gyrus.

When comparing the "same-syntax" condition to the "different-sentences" condition, no significant effect of adaptation emerged ( $p<.01$ voxel based) in the mask "sentence-fixation" contrast at $p<.001$. Contrasts between same syntax and different sentences restricted to each of the four types of constructions (active, passive, cleft, and cleft-passive) did not show any significant effect either. We also contrasted these four "same-syntax" conditions two by two, and did not observe any significant difference $(p<.01)$. 
Table 4. Peak Voxels of Activated Regions Showing Significant Adaptations $(p<.001$, Uncorrected Voxel-based; Clusters' Extent $>4$ Voxels; Masked by the "Sentence-Silence" Contrast at $p<.001$ )

\begin{tabular}{|c|c|c|c|c|c|c|}
\hline \multirow{2}{*}{$\begin{array}{l}\text { Adaptation Same Sentences > } \\
\text { Adaptation Different Sentences }\end{array}$} & \multirow[b]{2}{*}{ Z Score } & \multirow[b]{2}{*}{$p(F D R)$} & \multirow[b]{2}{*}{ Extent $\left(\mathrm{mm}^{3}\right)$} & \multicolumn{3}{|c|}{ MNI Coordinates } \\
\hline & & & & $x$ & $y$ & $z$ \\
\hline Left middle temporal gyrus & 5.04 & .002 & 465 & -54 & -45 & 3 \\
\hline Left middle temporal gyrus & 4.16 & .003 & 474 & -60 & -12 & -6 \\
\hline Left middle temporal gyrus & 3.93 & .004 & & -57 & -9 & -15 \\
\hline Left superior temporal gyrus & 3.8 & .005 & & -48 & 9 & -24 \\
\hline Left fusiform gyrus & 4.48 & .002 & 696 & -42 & -54 & -12 \\
\hline Left middle occipital gyrus & 4.23 & .003 & & -39 & -90 & -9 \\
\hline Left inferior occipital gyrus & 3.82 & .005 & & -45 & -75 & -12 \\
\hline Left inferior frontal gyrus pars triangularis & 3.88 & .004 & 120 & -42 & 21 & 6 \\
\hline Left inferior frontal gyrus pars opercularis & 3.57 & .006 & 75 & -42 & 6 & 27 \\
\hline Left inferior frontal gyrus pars opercularis & 3.46 & .007 & 30 & -60 & 18 & 24 \\
\hline Left supplementary motor area & 3.81 & .005 & 51 & -6 & -6 & 60 \\
\hline Left postcentral gyrus & 3.57 & .006 & 48 & -57 & -12 & 51 \\
\hline
\end{tabular}

\section{Discussion}

The reduction in number of syntactic constructions used in the experimental materials did not yield more syntactic adaptation than in the first experiment while the design was sensitive enough to detect clear adaptation effects due to the repetition of the exact same sentence. As mentioned in the Introduction, the published evidence for syntactic priming in comprehension is quite scarce. The occurrence of syntactic priming in speech production and in mixed perception-production experiments led us to expect syntactic priming in perception; yet, in a recent review, Branigan (2007) mentioned two behavioral experiments in perception where syntactic priming only occurred when the verb was repeated. The existence of syntactic priming and/or adaptation in comprehension is therefore not very well established.
For this reason, we decided to run a behavioral priming experiment modeled after Experiment 1.

\section{EXPERIMENT 3}

\section{Methods}

The experiment was identical in every respect to Experiment 1 . The only difference was that participants were seated in front of a computer rather than lying in the scanner and that they were asked to "press the response button at the end of each sentence once they had understood it." Response times, measured from the sentence ending, were collected by E-prime. As in the scanner, participants were asked to keep their eyes closed in the auditory condition.
Figure 4. Areas showing a significant decrease in activation across the four sentences in the "same-sentences" and the "same-syntax" conditions relative to the "differentsentences" condition $(p<.01$, uncorrected voxel-based).

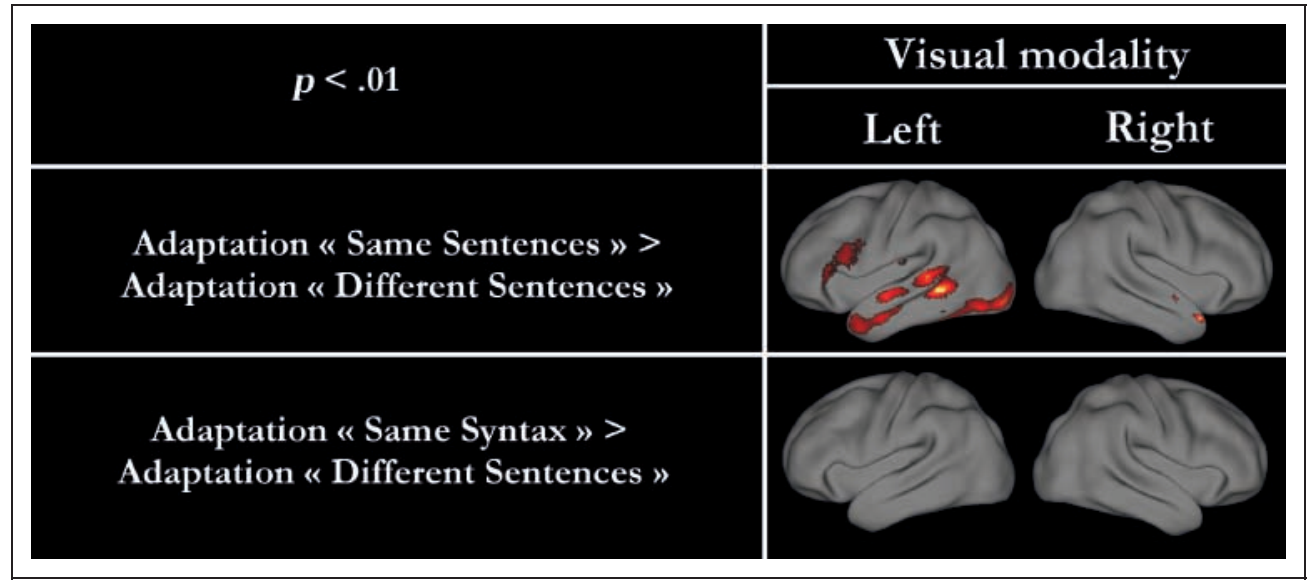




\section{Participants}

Twenty-six young adults (20 women and 6 men; age: mean $=21.8$ years; $S D=2.6$; range $=18-28$ years) participated in the experiment and received 10 euros. All were right-handed (scores at the Edinburgh test: mean = 76.1; $S D=18.6$; range $=50-100$ ) native speakers of French. The experiment was approved by the regional ethics committee, and written informed consent was obtained in all cases.

\section{Results}

Participants pressed on the button for $98.4 \%$ of the sentences in the visual modality and for $96.1 \%$ in the auditory modality. Median reaction times in each condition and for each sentence position were computed for each participant. Averaged data are displayed on Figure 5. The reaction times were quite fast because participants sometimes answered before the physical end of the sentence, anticipating it when reading or listening to the last word $(6.3 \%$ and $11.6 \%$ of the reaction times were negative in the visual and auditory conditions, respectively. The inclusion or replacement of these data points did not alter the conclusions of the following analyses). Analyses of variance revealed significant main effects of conditions and Condition $\times$ Position interactions for all pairs of conditions [all $p<.05$ except for the interaction "Same syntax" vs. "Different sentences" $\times$ Position in the auditory modality, where $F(3$, $75)=2.05, p=.11]$. Reaction times accelerated more in the "same-sentence" condition than in the "same lexicosemantic content" condition, more in the latter than in the "same-syntax" condition, and more in the "samesyntax" than in the "different-sentences" condition.

\section{Discussion}

When participants were asked to detect the end of the sentences, both "lexico-semantic" and "syntactic" priming were observed in the reaction times patterns. As in the fMRI experiment, priming by sentence content was the dominant effect, but syntactic priming, although about half as large, was also significant. Why was syntactic priming observed in behavioral but not in fMRI data? One obvious possibility is that fMRI was not sensitive enough to detect a small effect of syntactic adaptation. Another possibility is that the behavioral task induced participants to rely on peculiar strategies not present during fMRI, where the only task was to attend to meaning. In the same-syntax condition, it was possible to exploit the regularity of the syntactic structures, notably thanks to the repetition of the content words, in order to predict whether a given word would be the last of the sentence. When designing the fMRI experiments, we chose not to ask participants to perform an explicit decision task in order to measure activations related to the "normal," automatic processing of sentences. Whether
Figure 5. Mean reaction times (msec) to detect the end of the sentence according to condition and position.

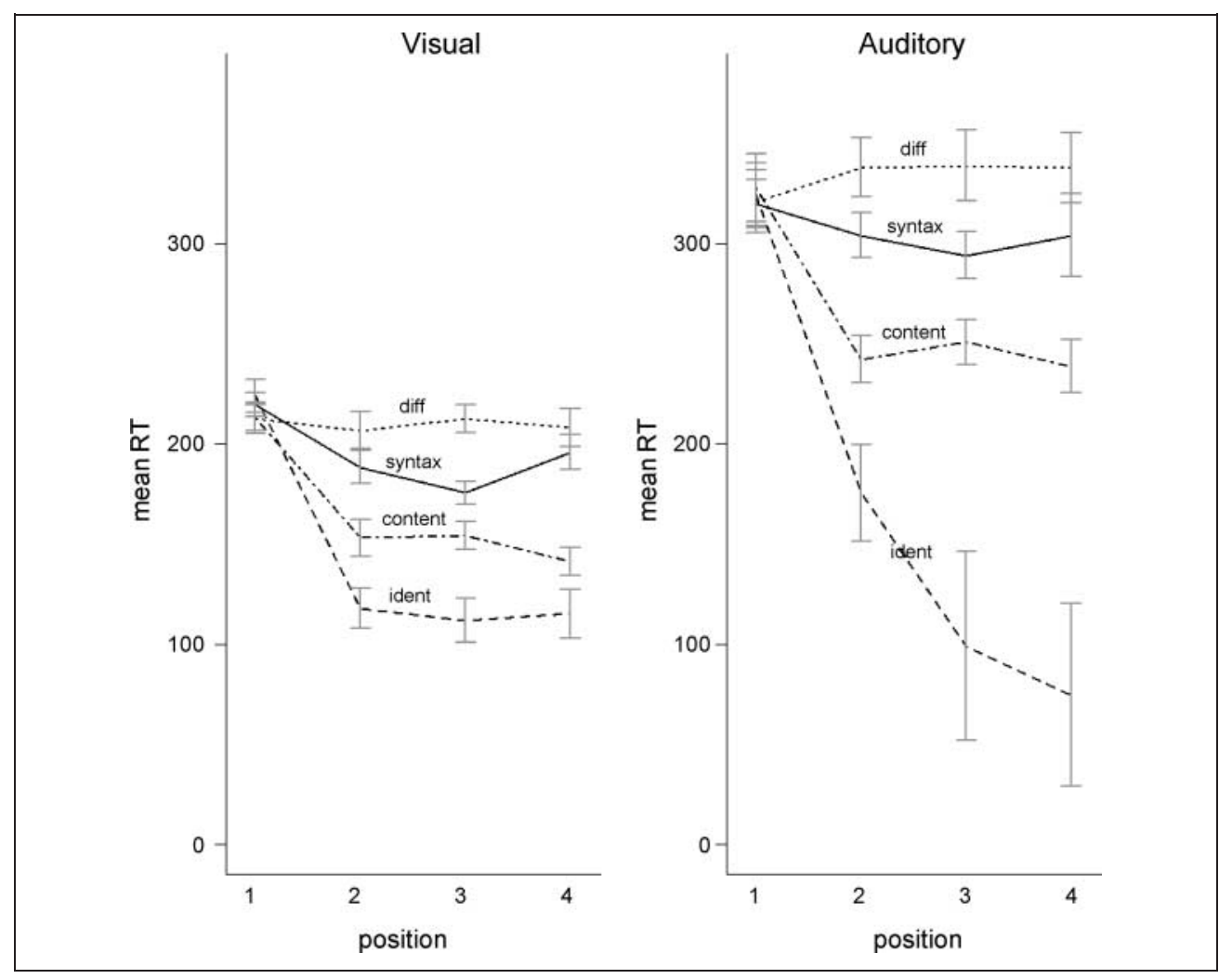


the contrasting results of the behavioral and the fMRI experiments are due to various task demands or to differential sensitivity is an open issue.

\section{GENERAL DISCUSSION}

In the first experiment, we observed reductions in the activity of several areas of the language network when a sentence was repeated. Relative to previous research, we have established two important new results: (1) the reduction in activation occurs for both visual and auditory sentence presentation, suggesting that many of the adaptation regions are coding for amodal levels of word, sentence, or semantic processing; (2) reduced activation can be observed even when sentences are not repeated identically, but when they share the same lexico-semantic content (i.e., they have the same content words and argument structure, but express different grammatical constructions). By contrast, there was no evidence for fMRI adaptation when successive sentences shared the same syntactic constructions but differed in lexico-semantic content. The second experiment, using a restricted set of four syntactic constructions, replicated this absence of significant syntactic adaptation. Yet, in a third purely behavioral experiment that used the design and material of Experiment 1, participants detected the end of sentences faster, both in syntactically and in semantically homogeneous blocks.

One possible interpretation could be that the "passive" reading or listening task did not force participants to fully parse the sentences (Bever, 1970). A similar argument was advanced by Indefrey, Hellwig, Herzog, Seitz, and Hagoort (2004). These authors found that activation was stronger in a dorsal part of Broca's area when participants produced full-fledged sentences rather than simpler word lists. However, when listening to the same utterances, there was no difference in brain activation between full sentences and word lists. This finding led the authors to propose that the degree to which listeners recruit syntactic processing resources in language comprehension may be a function of the syntactic demands of the task or the stimulus material. Our participants were not forced to engage in a syntactic analysis of the stimuli as they just had to read or listen to the sentences passively. The same applies to the participants in Noppeney and Price's study, but the presence of locally ambiguous and garden-path sentences may have induced them to engage more in syntactic computations.

The presence of syntactic priming in the reaction time data and its absence in fMRI data may be due to a lower sensitivity of the fMRI technique. Alternatively, as mentioned in the discussion of Experiment 3, the drop in reaction times might be due to specific strategies induced by task demands. Therefore, one cannot exclude the possibility that the absence of syntactic fMRI adaptation in comprehension is genuine and reveals some- thing significant on the computational building blocks in parsing. Our experiment intended to find out whether the repetition of a grammatical construction provokes a reduction of activity in brain areas encoding linguistic representations. A construction is a kind of a structural schema, or skeleton, defined by certain elements of the functional lexicon and the subtrees and computational processes associated to them. For instance, each cleft construction has the It is ... that ... configuration and involves $A^{\prime}$ movement of the clefted element, each passive has be auxiliary and past participial morphology, and involves "A" movement of the object to subject position, each question has a Wh element and inversion, and involves " $\mathrm{A}$ " movement of the Wh element, and so forth. According to current linguistic analyses, a construction is not a primitive structural object, but a conglomerate of such finer properties: choices in the functional lexicon, elementary subtrees, and elementary computational processes such as the different kinds of movement. The lack of adaptation may then be taken as an indication that the parser does not build construction-based representations, but directly attends the finer computational ingredients just mentioned.

In contrast to the absence of syntactic adaptation in fMRI data, we found clear adaptation effects when sentences having the same lexico-semantic content were repeated. In particular, regions of the left superior and middle temporal gyri bordering the STS showed reduced activation. These regions are prime candidates for the processing of sentence meaning (Davis \& Johnsrude, 2003; Friederici et al., 2003). Although our design does not allow us to properly disentangle the contributions of word forms, word meanings, argument structure, or whole sentence meaning to the repetition effects, it is interesting to note that adaptation was observed, for the visual modality only, in the fusiform gyrus in the vicinity of the VWF, where Cohen et al. (2004) have described adaptation when isolated words were repeated. Our results confirm and extend their finding to words presented within sentences. The repetition of lexico-semantic content, in the reading task only, also yielded adaptation effects in the left inferior frontal and precentral regions. The precise nature of the processes involved cannot be established with certainty but it is noteworthy that Klein et al. (2006) and Chee, Soon, and Lee (2003) have described adaptation effects in the frontal regions (BA 44, BA 45, and BA 9) when a word is followed by its translation in another language.

It is interesting to compare our study with another one that looked for modality-specific and common regions activated by passive reading and by passive listening (Jobard, Vigneau, Mazoyer, \& Tzourio-Mazoyer, 2007). These authors reported common activations in the bilateral precentral and inferior frontal regions, and in the left temporal gyri, as well as in the posterior part of the middle temporal gyrus, extending ventrally in the occipito-temporal junction. In the present study, the 
intersection of regions activated by reading or listening (significantly more than during the silent baseline) belonged exclusively to the left hemisphere and comprised most of the middle and superior temporal gyrus, the inferior frontal regions (pars triangularis and pars opercularis), as well as areas in the precentral gyrus and in the supplementary motor area. The frontal activations are quite similar to that described by Jobard et al. (2007). However, the temporal activations that we observed are more extensive. This is likely due to the fact that some of the stimuli used by Jobard et al. were lists of words, which yield less activation than sentences in anterior temporal regions. One unexplained discrepancy between Jobard et al.'s results and ours is the absence of activation in the basal temporal language area, or in the VWFA, during the auditory stimulation in the present study.

Disentangling the contributions of the different levels of representation involved in the adaptation observed when a sentence is repeated remains an avenue for future research. Grammatical constructions are complex objects consisting of conglomerates of more elementary computational ingredients, and it is conceivable that syntactic priming in comprehension only occurs for some of those computational elements. For instance, systematically contrasting sentence types involving A versus A-bar movements is a possibility. If one could separate the contributions of lexical and sentence level meaning, this would also be worth investigating.

\section{Acknowledgments}

This research was supported by a grant from the NEST Pathfinder program of the European Union ("Neurocom" project; contract 12738). We thank D. Le Bihan, G. DehaeneLambertz, and A. Jobert.

Reprint requests should be sent to Anne-Dominique Devauchelle and Christophe Pallier, Inserm U562, CEA/SAC/DSV/DRM/ Neurospin, Batiment 145, Point Courrier 156, F-91191 Gif/Yvette Cedex, France, or via e-mail: ad_devauchelle@yahoo.fr and christophe.pallier@cea.fr.

\section{REFERENCES}

Bever, T. (1970). The cognitive basis for linguistic structures. In J. R. Hayes (Ed.), Cognition and the development of language (pp. 279-360). New York: Wiley.

Bock, J. K. (1986). Syntactic persistence in language production. Cognitive Psychology, 18, 355-387.

Bock, J. K., Dell, G. S., Chang, F., \& Onishi, K. H. (2007). Persistent structural priming from language comprehension to language production. Cognition, 104, 437-458.

Branigan, H. P. (2007). Syntactic priming. Language and Linguistics Compass, 1, 1-16.

Branigan, H. P., Pickering, M. J., \& McLean, J. F. (2005). Priming prepositional-phrase attachment during comprehension. Journal of Experimental Psychology: Learning, Memory, and Cognition, 31, 468-481.

Branigan, H. P., Pickering, M. J., Stewart, A. J., \& McLean, J. F. (2000). Syntactic priming in spoken production: Linguistic and temporal interference. Memory $\&$ Cognition, 28, 1297-1302.

Caplan, D., Alpert, N., \& Waters, G. (1999). PET studies of syntactic processing with auditory sentence presentation. Neuroimage, 9, 343-351.

Chee, M. W. L., Soon, C. S., \& Lee, H. L. (2003). Common and segregated neuronal networks for different languages revealed using functional magnetic resonance adaptation. Journal of Cognitive Neuroscience, 15, 85-97.

Cohen, L., Jobert, A., Bihan, D. L., \& Dehaene, S. (2004). Distinct unimodal and multimodal regions for word processing in the left temporal cortex. Neuroimage, 23, $1256-1270$.

Davis, M. H., \& Johnsrude, I. S. (2003). Hierarchical processing in spoken language comprehension. Journal of Neuroscience, 23, 3423-3431.

Dehaene-Lambertz, G., Dehaene, S., Anton, J.-L., Campagne, A., Ciuciu, P., Dehaene, G. P., et al. (2006). Functional segregation of cortical language areas by sentence repetition. Human Brain Mapping, 27, 360-371.

Frazier, L., Taft, L., Roeper, T., \& Clifton, C. (1984). Parallel structure: A source of facilitation in sentence comprehension. Memory \& Cognition, 12, 421-430.

Friederici, A. D. (2002). Towards a neural basis of auditory sentence processing. Trends in Cognitive Sciences, 6, 78-84.

Friederici, A. D., Meyer, M., \& von Cramon, D. Y. (2000). Auditory language comprehension: An event-related fMRI study on the processing of syntactic and lexical information. Brain and Language, 75, 289-300.

Friederici, A. D., Rüschemeyer, S.-A., Hahne, A., \& Fiebach, C. J. (2003). The role of left inferior frontal and superior temporal cortex in sentence comprehension: Localizing syntactic and semantic processes. Cerebral Cortex, 13, 170-177.

Grill-Spector, K., \& Malach, R. (2001). fMRI-adaptation: A tool for studying the functional properties of human cortical neurons. Acta Psychologica, 107, 293-321.

Grodzinsky, Y., \& Friederici, A. D. (2006). Neuroimaging of syntax and syntactic processing. Current Opinion in Neurobiology, 16, 240-246.

Hasson, U., Nusbaum, H. C., \& Small, S. L. (2006). Repetition suppression for spoken sentences and the effect of task demands. Journal of Cognitive Neuroscience, 18, 2013-2029.

Indefrey, P., Hagoort, P., Herzog, H., Seitz, R. J., \& Brown, C. M. (2001). Syntactic processing in left prefrontal cortex is independent of lexical meaning. Neuroimage, 14, 546-555.

Indefrey, P., Hellwig, F., Herzog, H., Seitz, R. J., \& Hagoort, P. (2004). Neural responses to the production and comprehension of syntax in identical utterances. Brain and Language, 89, 312-319.

Jobard, G., Vigneau, M., Mazoyer, B., \& Tzourio-Mazoyer, N. (2007). Impact of modality and linguistic complexity during reading and listening tasks. Neuroimage, 34, 784-800.

Just, M. A., Carpenter, P. A., Keller, T. A., Eddy, W. F., \& Thulborn, K. R. (1996). Brain activation modulated by sentence comprehension. Science, 274, 114-116.

Kaan, E., \& Swaab, T. Y. (2002). The brain circuitry of syntactic comprehension. Trends in Cognitive Sciences, 6, 350-356.

Klein, D., Zatorre, R. J., Chen, J.-K., Milner, B., Crane, J., Belin, P., et al. (2006). Bilingual brain organization: A functional magnetic resonance adaptation study. Neuroimage, 31, 366-375.

Levelt, W., \& Kelter, S. (1982). Surface form and memory in question answering. Cognitive Psychology, 14, 78-106.

Mazoyer, B. M., Dehaene, S., Tzourio, N., Frak, V., Murayama, N., Cohen, L., et al. (1993). The cortical representation of speech. Journal of Cognitive Neuroscience, 5, 467-479. 
Mehler, J., \& Carey, P. (1967). Role of surface and base structure in the perception of sentences. Journal of Verbal Learning and Verbal Behavior, 6, 335-338.

Mériaux, S., Kherif, F., Pallier, C., Izard, V., Brett, M., Garnero, L., et al. (2003). Assessing the homogeneity of subject's samples for random effect analyses in fMRI. In Proceedings of 9 th OHBM Meeting. New York: OHBM.

Naccache, L., \& Dehaene, S. (2001). The priming method: Imaging unconscious repetition priming reveals an abstract representation of number in the parietal lobes. Cerebral Cortex, 11, 966-974.

Newman, A. J., Pancheva, R., Ozawa, K., Neville, H. J., \& Ullman, M. (2001). An event-related fMRI study of syntactic and semantic violations. Journal of Psycholinguistics Research, 30, 339-364.

Noppeney, U., \& Price, C. J. (2004). An fMRI study of syntactic adaptation. Journal of Cognitive Neuroscience, $16,702-713$.

Osterhout, L., \& Holcomb, P. (1992). Event-related brain potentials elicited by syntactic anomaly. Journal of Memory and Language, 31, 785-806.

Pickering \& Branigan (1999). Syntactic priming in language production. Trends in Cognitive Sciences, 3, 136-141.

Potter, M. C., \& Lombardi, L. (1998). Syntactic priming in immediate recall of sentences. Journal of Memory and Language, 38, 265-282.

Smith, M., \& Wheeldon, L. (2001). Syntactic priming in spoken sentence production-An online study. Cognition, 78, $123-164$.

Stromswold, K., Caplan, D., Alpert, N., \& Rauch, S. (1996). Localization of syntactic comprehension by positron emission tomography. Brain and Language, 52, 452-473. 